\title{
ANALYSE COMPARATIVE DES TEMPÉRATURES EXTRÊMES DE 1950 A 2100 ISSUES DES MODÈLE ARPEGE-CLIMAT ET LMD : INTÉRÊT POUR L'ACTIVITÉ VITICOLE CHAMPENOISE
}

\author{
Elodie BRICHE ${ }^{1}$, Malika MADELIN ${ }^{1}$, Gérard BELTRANDO ${ }^{1,2}$ \\ et Claude KERGOMARD ${ }^{3}$ \\ ${ }^{1}$ UMR 8586 (PRODIG) \\ C.C. 7001, Université Paris Diderot (Paris VII), \\ 75205 Paris cedex 13 \\ elodie.briche@univ-paris-diderot.fr \\ ${ }^{2}$ Laboratoire COSTEL (en détachement contractuel 2009-10) \\ UMR 6554 CNRS, LETG, Université de Rennes 2 \\ Place du Recteur Henri Moal \\ 35043 Rennes Cedex \\ ${ }^{3}$ Ecole Normale Supérieure - Département de Géographie \\ 48 , boulevard Jourdan \\ 75014 Paris
}

\begin{abstract}
Résumé
Dans un contexte de changement climatique global, l'évaluation des modifications engendrées sur des échelles fines telles que l'agrosystème viticole champenois s'avère nécessaire, notamment en termes de fréquences des extrêmes thermiques. Ces derniers influencent le bon déroulement des cycles végétatif et productif de la vigne. Cet article traite de l'analyse comparative des séries de températures minimales et maximales journalières issues de quatre points de grille du modèle ARPEGE-Climat, d'un point de grille du modèle du LMD et d'une station de référence du vignoble. Une période de contrôle (1950-2000) permet de mettre en évidence les biais froids des distributions statistiques des sorties de modèles au printemps et en été, notamment en termes d'estimation de fréquences d'extrêmes. Les sorties de modèles des points de grille retenus sont ensuite utilisées pour évaluer l'évolution, jusqu'en 2100, de ces extrêmes climatiques pour trois scénarios prenant en compte les changements socio-économiques du monde. Conformément aux conclusions du GIEC (2007), la fréquence des extrêmes chauds estivaux dans le futur devrait augmenter tandis que les extrêmes froids d'avril, au moment du débourrement, devraient diminuer. Cependant, dans un contexte de changement climatique, le débourrement pourrait être plus précoce et des occurrences d'extrêmes froids printaniers en mars pourraient provoquer un gel accru des bourgeons.
\end{abstract}

Mots-clés : modèles du climat, changement climatique, vignoble de Champagne, températures extrêmes journalières.

\section{Abstract \\ Extreme temperatures comparative analysis (1950 to 2100) of ARPEGE-Climate and LMD models: interest for vineyard activity}

In the context of global warming, modifications assessment at fine scales as Champagne agrosystem is necessary in terms of extremes frequency because extremes influence vegetative cycle of vineyard. This study deals with comparative analysis between four ARPEGE-Climate grid points, one LMD grid point and one reference vineyard station minimal and maximal daily temperatures. A control period (1950-2000) underlines cold biases of temperatures statistical distributions in spring and summer. Grid point temperatures are used to evaluate extremes evolution, until 2100, in function of three scenarios, which take economical and social changes into account. Indeed, extreme hazards frequency can increase in the future. In accordance with IPCC conclusions, hot summer extremes could increase in the future while cold spring extremes could decrease during budbreak. However, in the context of climate change, budbreak could be earlier and spring cold extremes in March could provoke more severe frost of buds.

Keywords: climate models, climate change, Champagne vineyard, extreme daily temperatures.

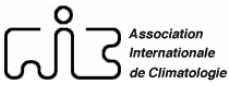




\section{Introduction}

Les premiers constats du changement climatique sont déjà observables sur les agrosystèmes et notamment sur la viticulture. Ils engendrent ainsi de multiples interrogations sur les impacts futurs sur ces agrosystèmes et leurs conséquences économiques (Seguin, 2004 ; Bindi et Howden, 2004 ; Jones et al., 2005a et b ; Jones, 2006 ; Jones, 2007). Au niveau local, elles conduisent à rendre nécessaire une évaluation à l'échelle du terroir afin de mettre en place une adaptation aux modifications du climat moyen, ainsi qu'aux fréquences d'évènements extrêmes dommageables pour l'économie vini-viticole tels que le gel de printemps. Par définition, les extrêmes sont des phénomènes rares, intenses, à l'origine de pertes socio-économiques importantes (Beniston et al., 2007). Des changements dans l'intensité et la fréquence des extrêmes climatiques pourraient avoir des conséquences environnementales et socio-économiques (Kharin et Zwiers, 2000). Dans le cas de la vigne, les périodes clés du cycle pour la vulnérabilité aux extrêmes thermiques sont bien identifiées : au printemps, pendant le débourrement après la période de dormance et lors de la maturation, durant la période estivale. Dans un contexte de réchauffement avéré, l'étude des conditions thermiques futures est essentielle pour aider les professionnels à répondre aux questions d'adaptation des pratiques culturales. Selon le dernier rapport du GIEC (2007) et d'après les résultats des modèles de circulation générale (MCG), il devrait y avoir dans le futur une fréquence plus importante d'extrêmes chauds et une fréquence plus faible d'extrêmes froids, associées à un climat moyen plus chaud. La confiance dans la capacité de ces modèles à prévoir le climat futur a augmenté (entre les rapports du GIEC de 2001 et 2007). Les progrès en matière de modélisation permettent d'obtenir des sorties à résolution régionale telles que, dans le contexte français, celles des modèles ARPEGE-Climat (Retic) de Météo-France (résolution $50 \mathrm{~km}$ ) et du Laboratoire de Météorologie Dynamique (300 km).

Dans cette étude, les températures des sorties des modèles ARPEGE-Climat et LMD sont confrontées et comparées, en termes de distribution statistique et de fréquences d'extrêmes à celles d'une station de référence, Reims-Courcy (située à proximité du vignoble champenois) sur une période passée dite "de contrôle» (1950-2000). Différents scénarios climatiques futurs (2001-2100) sont ensuite analysés afin de rendre compte des modifications thermiques possibles futures, en axant ici l'analyse sur les extrêmes chauds estivaux et gélifs printaniers.

\section{Données et méthodes}

\subsection{Reims-Courcy, station de référence à proximité du vignoble}

Les températures minimales et maximales journalières de la station de référence ReimsCourcy (figure 1) sur la période 1950-2000 sont utilisées. Cette station dispose d'une longue série de données fiables fournies par Météo-France dont l'homogénéité a été testée au préalable (Pettitt, 1979) sur la période dite " de contrôle »1950-2000. Une rupture est détectée en 1987 sur la série des températures moyennes annuelles. Il s'agit d'une rupture statistiquement significative $(\alpha=5 \%)$ probablement d'origine climatique car elle ne résulte pas d'un changement de capteur ou d'un déplacement de la station; cette rupture de 1987-88 est d'ailleurs observée dans un grand nombre de stations météorologiques françaises (Moisselin et al., 2002). La station de Reims-Courcy, située en plaine à $95 \mathrm{~m}$ est considérée comme représentative des conditions thermiques du vignoble marnais.

\subsection{Les sorties du modèle ARPEGE-Climat (Retic) et LMD}

Les deux modèles couplés français sont utilisés afin de déterminer les sorties thermiques les plus adaptées au type d'étude envisagé. Leurs différences majeures résident dans la 
représentation de leur dynamique atmosphérique (Dufresne et Royer, 2007) et dans leurs résolutions: les mailles du modèle ARPEGE-Climat sont plus fines que celles du LMD (respectivement de 50 et $300 \mathrm{~km}$ de résolution) et correspondent plus à l'échelle d'un vignoble.

Figure 1: Zone étudiée avec le vignoble marnais, les points de grille ARPEGE-Climat et la station de référence Reims-Courcy (données : SRTM et BD Carto IGN fournie par le CIVC, projection Lambert 2 étendu). Study area: Champagne vineyard, points of ARPEGEClimate model and Reims-Courcy station (data of CIVC: SRTM and BD Carto IGN, Lambert 2 projection).

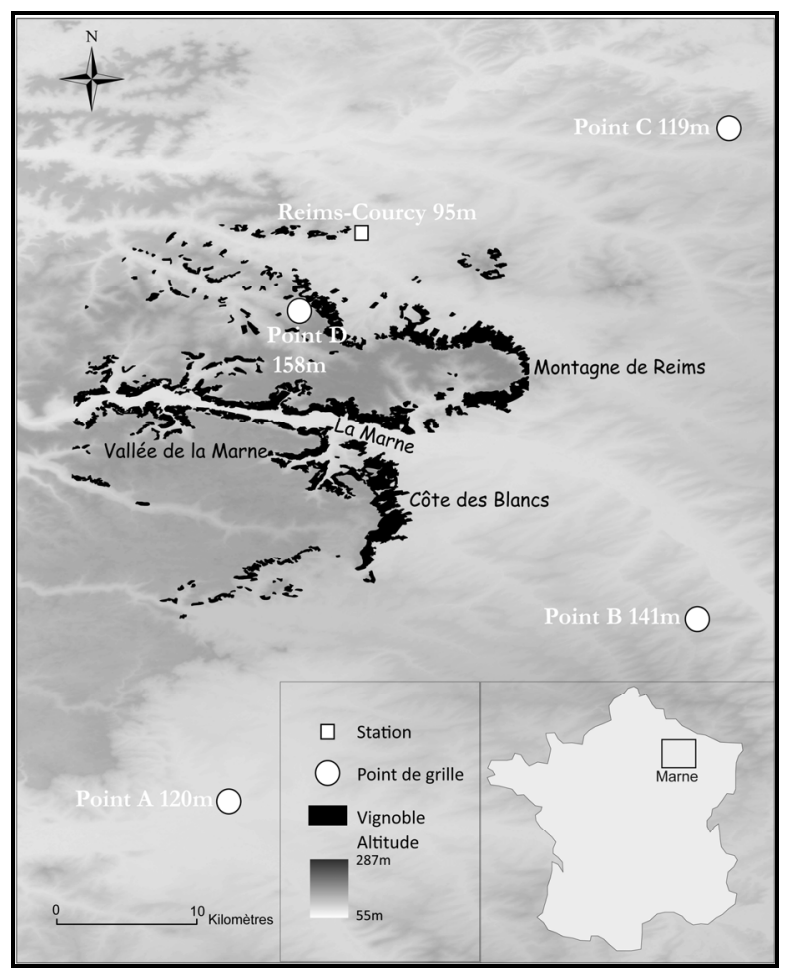

Les températures journalières minimales et maximales (30 jours par mois) du point de grille du modèle LMD (Institut Pierre Simon Laplace) le plus proche du vignoble sont extraites sur la période de contrôle (1950-2000) et sur trois scénarios de simulations futures (2001-2100). La résolution horizontale de ce modèle est d'environ $2^{\circ}$ en longitude et $2^{\circ}$ en latitude pour les moyennes latitudes, soit environ une maille de $300 \mathrm{~km}$ de côté. Ces sorties sont en deux dimensions et ne tiennent pas compte de l'altitude (Salas-Mélia et al., 2005 ; Dufresne et al., 2006). Ce point de grille recouvre le vignoble marnais.

Les sorties ARPEGE-Climat (Retic) sont issues de la version 4 du modèle de circulation générale tridimensionnel, incluant l'altitude. La maille variable a permis de développer des études régionales du climat (Déqué et Piedelievre, 1995 ; Gibelin et Déqué, 2003) avec une résolution d'environ $50 \mathrm{~km}$ pour la France métropolitaine.

Pour les simulations passées et futures sont retenues les données thermiques journalières (minimales et maximales) des sorties du modèle ARPEGE-Climat de 1950 à 2100 de quatre points de grille du modèle englobant le vignoble marnais (figure 1).

\subsection{Les scénarios du GIEC choisis}

Nous avons choisi les trois scénarios de simulations du climat futur les plus utilisés actuellement dans les études d'impacts (Garcia de Cortazar, 2006 ; Lauffenburger, 2007) : les scénarios A1B, A2 et B1 (tableau 1). La période de contrôle (1950-2000) permet la comparaison entre les points de grille du modèle représentant la température d'une maille et les températures de la station de référence Reims-Courcy. Cette période ne tient pas compte des données observées : les températures sont calculées par le modèle sans prise en compte des observations (Dufresne et al., 2006). 


\begin{tabular}{|c|c|}
\hline & $\begin{array}{l}\text { - Le canevas et la famille de scénarios A1 décrivent un monde futur dans lequel la croissance } \\
\text { économique sera très rapide, la population mondiale atteindra un maximum au milieu du siècle pour } \\
\text { décliner ensuite et de nouvelles technologies plus efficaces seront introduites rapidement. } \\
\text { - Le scénario A1B se caractérise par un équilibre entre les sources ce qui signifie que l'on ne s'appuie } \\
\text { pas excessivement sur une source d'énergie particulière, en supposant que des taux d'amélioration } \\
\text { similaires s'appliquent à toutes les technologies de l'approvisionnement énergétique et des utilisations } \\
\text { finales. }\end{array}$ \\
\hline A2 & $\begin{array}{l}\text { - Le canevas et la famille de scénarios A2 décrivent un monde très hétérogène. Les schémas de } \\
\text { fécondité entre régions convergent très lentement, avec pour résultat un accroissement continu de la } \\
\text { population mondiale. Le développement économique a une orientation principalement régionale. }\end{array}$ \\
\hline B1 & $\begin{array}{l}\text { - Le canevas et la famille de scénarios B1 décrivent un monde convergent avec la même population } \\
\text { mondialc culminant au milicu du sic̀cle ct déclinant cnsuitc avec des changements rapides dans les } \\
\text { structures économiques vers une économie de services et d'information, avec des réductions dans } \\
\text { l'intensité des matériaux et l'introduction de technologies propres et utilisant les ressources de } \\
\text { manière efficiente. L'accent est sur des solutions mondiales orientées vers une viabilité économique, } \\
\text { sociale et environnementale, y compris une meilleure équité, mais sans initiatives supplémentaires } \\
\text { pour gérer le climat. }\end{array}$ \\
\hline
\end{tabular}

Tableau 1 : Récapitulatif des scénarios de simulations futures utilisés dans le cadre de l'étude (adapté de Nakicenovic et Swart, 2000). Summary of future simulations used to in the study (adapted to Nakicenovic and Swart, 2000).

\subsection{Analyse comparative des extrêmes thermiques}

Les extrêmes thermiques sont étudiés et comparés sur la période de contrôle de 1950 à 2000, puis on considère l'évolution future de ces extrêmes de 2001 à 2100 pour les deux modèles sur les trois scénarios retenus.

\subsubsection{Seuils thermiques et extrêmes nuisibles à la vigne}

Par définition, les extrêmes se produisent très souvent à une résolution spatiale fine, d'où la difficulté de les modéliser précisément et l'importance de les traiter dans des études de comparaison modèle/station (Palutikof et al., 1997). Ces phénomènes rares sont souvent, d'un point de vue statistique, en-dessous du $1^{\text {er }}$ décile et au-dessus du $9^{\text {ème }}$ décile des distributions de températures (Beniston et al., 2007), voire même en dessous du $5^{\text {ème }}$ centile et au-dessus du $95^{\text {ème }}$ centile (Kharin et Zwiers, 2000). Les extrêmes, valeurs à fréquence faible ou de faible amplitude, diminuent graduellement en deux queues (inférieure et supérieure) qui entourent les valeurs à grandes fréquences.

Dans cette étude, les extrêmes dommageables pour la vendange en qualité et quantité sont mis en évidence en observant et en comparant, sur la période de contrôle, les distributions statistiques des sorties des modèles à la mesure sur la station de référence aux deux périodes clés identifiées pour la vigne : au moment de l'aléa gélif printanier (avril) et durant la période estivale de maturation des baies.

Ainsi, deux seuils caractéristiques sont étudiés :

1) Le seuil estival de $35^{\circ} \mathrm{C}$ au-delà duquel les températures extrêmes favorisent l'échaudage des baies. En effet, la bibliographie montre que le seuil estival se situe entre 35 et $40^{\circ} \mathrm{C}$ (Champagnol, 1984 ; Huglin et Schneider, 1998). Dokoolzian et Bergqvist (2001) ont montré que des températures supérieures à $37^{\circ} \mathrm{C}$ limitent le remplissage des baies. Les températures supérieures à $35^{\circ} \mathrm{C}$ pendant la phase de maturation perturbent également la synthèse des flavanoïdes qui contribuent à la bonne synthèse des tanins 
(Matus et al., 2009). Nous avons donc retenu le seuil de $35^{\circ} \mathrm{C}$ et détaillé la queue supérieure des distributions statistiques estivales.

2) $\mathrm{Au}$ printemps, des températures inférieures à $-2^{\circ} \mathrm{C}$, observées sur la queue inférieure des distributions statistiques des températures journalières d'avril, au moment du débourrement, peuvent provoquer le gel des bourgeons floraux et compromettre le développement des baies (Huglin et Schneider, 1998 ; Madelin, 2004) : il s'agit du gel agronomique. Les gelées météorologiques sont radiatives ou advectives; dans la réalité, le risque gélif résulte de la combinaison des deux phénomènes associée à la vulnérabilité du végétal.

Sur la période de simulation future (2001-2100) des deux modèles, on étudie les fréquences probables d'aléas extrêmes et les tendances sur les deux périodes clés pour la vigne retenues avec les trois scénarios futurs.

\subsubsection{Tests statistiques et extrêmes thermiques journaliers}

Les distributions d'extrêmes chauds estivaux et froids printaniers sur la période de contrôle ne suivent pas une loi normale et ce, de manière statistiquement significative à $5 \%$ d'après les résultats du test d'Anderson-Darling ${ }^{1}$, utilisé en préalable de l'utilisation du test non paramétrique suivant. Le test de Kolmogorov-Smirnov (Siegel et Castellan, 1988; Williams, 2001) permet de comparer les distributions de chaque sortie de modèle à la distribution de la station Reims-Courcy et de déterminer si elles suivent la même loi de manière statistiquement significative (avec alpha $=5 \%$ ). L'hypothèse nulle ( $\mathrm{H} 0$ : les deux distributions suivent une même loi) est rejetée si la valeur critique de la table associée est inférieure à la valeur du test observée sur les données. Ce test est souvent utilisé dans les études sur les extrêmes hydro-climatiques en préalable de la réalisation de loi de Gumbel qui permet de calculer la période de retour d'évènements extrêmes (Kharin et Zwiers, 2000). Il s'agit d'un test d'hypothèse non paramétrique : il n'y a aucune hypothèse sur la distribution sous-jacente des données. Ici, les échantillons étudiés (modèle/station) sont indépendants. Le test de Kolmogorov-Smirnov convient lorsque les distributions ne sont pas normales, présentent des queues allongées et généralement dissymétriques. Ces caractéristiques sont observées sur les périodes clés étudiées.

\section{Comparaison des températures extrêmes entre les sorties de modèles et Reims-Courcy (1950-2000)}

Pour savoir si les sorties de modèles thermiques journalières peuvent être directement utilisées dans les études d'impacts, des comparaisons avec des données observées (ReimsCourcy) sont effectuées au moyen de statistiques sommaires et les formes de distributions sont comparées sur les périodes clés du cycle végétatif de la vigne.

\subsection{Des extrêmes estivaux dommageables peu fréquents}

Le test de Kolmogorov-Smirnov montre que les distributions statistiques des sorties de modèles (figure 2), qui ont des allures assez similaires avec un décalage plus prononcé vers la

\footnotetext{
${ }^{1}$ Le test d'Anderson-Darling est utilisé comme test de normalité afin de justifier l'utilisation du test nonparamétrique précédent. Ce test (Stephens, 1974) permet de vérifier si une distribution suit une loi normale. Les valeurs critiques de ce test sont dépendantes de la loi testée. Il s'agit d'un test d'hypothèse. L'hypothèse nulle (H0 : la distribution suit une loi normale) est rejetée si le test statistique donne une valeur plus grande que la valeur critique de la table associée.
} 
droite pour la courbe LMD, ne suivent pas les mêmes distributions que la station, de manière statistiquement significative à 5\%.

Figure 2 : Distributions statistiques des températures maximales journalières en juin, juillet et août pour Reims-Courcy et les points de grille de 1950 à 2000 (données CIVC, LMD et Météo-France - CNRM). Statistical distributions of daily maximum temperatures (June, July and August) for ReimsCourcy and grid points from 1950 to 2000 (data from CIVC, LMD and Météo-France-CNRM).

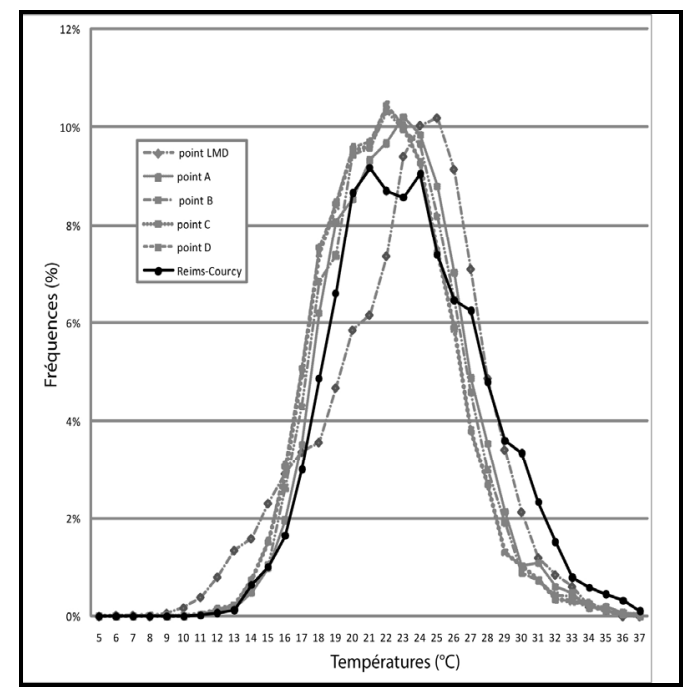

Sur les queues supérieures de distributions, le maximum de la station Reims-Courcy est de $37,7^{\circ} \mathrm{C}$ contre $34,8^{\circ} \mathrm{C}$ pour le modèle LMD et $36,7^{\circ} \mathrm{C}$ pour le point $\mathrm{B}$ : un biais froid entre 1 et $2^{\circ} \mathrm{C}$ pour le modèle ARPEGE-Climat selon le point de grille et de $3^{\circ} \mathrm{C}$ pour le modèle LMD est observé sur les extrêmes chauds estivaux. En termes de fréquences par rapport au seuil de $35^{\circ} \mathrm{C}$, les températures extrêmes pour le modèle ARPEGE-Climat cumulent entre $0,15 \%$ (points $\mathrm{C}$ et $\mathrm{D}$ ) et $0,30 \%$ (point $\mathrm{A}$ ) d'extrêmes supérieurs ou égaux à $35^{\circ} \mathrm{C}, 0,13 \%$ pour le point de grille LMD contre $0,87 \%$ pour Reims-Courcy : les sorties de modèles présentent un biais froid pour les extrêmes estivaux. Ce biais froid est également constaté sur les queues inférieures des distributions des sorties de modèles : le minimum de Reims-Courcy est de $10,5^{\circ} \mathrm{C}$ tandis que celui du point LMD est de $5,8^{\circ} \mathrm{C}$ avec un biais de $4,7^{\circ} \mathrm{C}$ et les minimas des points de grille du modèle ARPEGE-Climat varient entre $8^{\circ} \mathrm{C}$ (point $\mathrm{D}$ ) et $8,5^{\circ} \mathrm{C}$ (point $\mathrm{C}$ ), avec un biais sur le minimum d'environ $2^{\circ} \mathrm{C}$.

\subsection{Des aléas gélifs printaniers redoutés}

Les allures des distributions des cinq sorties de modèles et de Reims-Courcy (figure 3) sont assez similaires, pourtant celles des modèles ne suivent pas la même distribution que la station (au seuil de Kolmogorov-Smirnov à 5\%).

Figure 3 : Distributions statistiques des températures minimales journalières en avril pour Reims-Courcy et les points de grille de 1950 à 2000 (données CIVC, LMD et Météo-France - CNRM). Statistical distributions of daily minimal temperatures (April) for Reims-Courcy and grid points from 1950 to 2000 (data from CIVC, LMD and Météo-France CNRM).

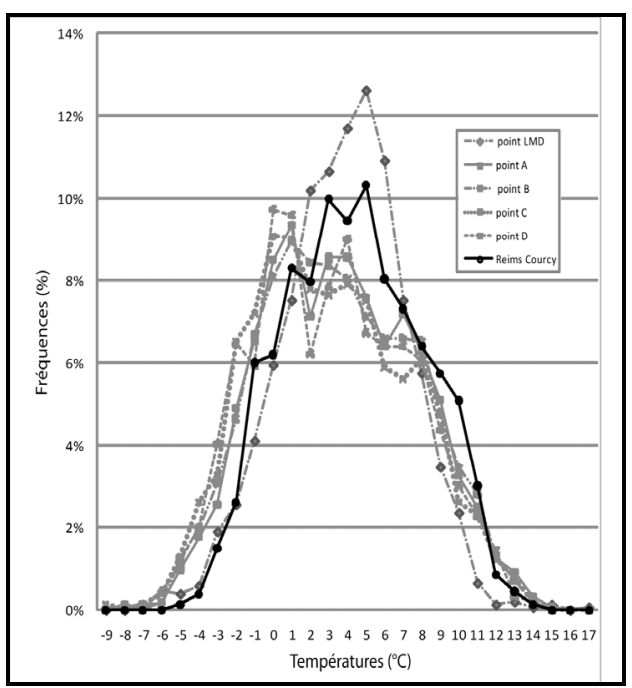


La moyenne des températures journalières d'avril de Reims-Courcy sur la période de contrôle est de $3,8^{\circ} \mathrm{C}$; elle est plus chaude de $0,6^{\circ} \mathrm{C}$ (LMD) à $1,2^{\circ} \mathrm{C}$ (point $\mathrm{D}$ ) par rapport aux sorties de modèles. Les sorties de modèles présentent un biais froid évident corroboré par les queues inférieures des distributions qui mettent en valeur les extrêmes gélifs printaniers (i.e. $<-2^{\circ} \mathrm{C}$ ) : le minimum de Reims-Courcy est de $-5,5^{\circ} \mathrm{C}$ contre $-9,2^{\circ} \mathrm{C}$ (Point D) et $-7,5^{\circ} \mathrm{C}$ (LMD). Les sorties de modèles sur la queue inférieure sont entre $3,7^{\circ} \mathrm{C}$ (point D) et $-2^{\circ} \mathrm{C}$ (LMD) plus froides que la station de référence à proximité du vignoble, elle-même considérée comme froide par rapport à l'ensemble du vignoble lors des gelées printanières (Madelin, 2004). Les distributions montrent que les fréquences égales à $-2^{\circ} \mathrm{C}$ font partie des grandes fréquences : les courbes s'affinent et les queues inférieures apparaissent autour de $-5^{\circ} \mathrm{C}$. En avril, les fréquences de températures inférieures et égales au seuil de $-2^{\circ} \mathrm{C}$ sont presque triplées pour les points de grille $\mathrm{C}$ et $\mathrm{D}(14 \%$ pour chaque point en cumul) par rapport à la station Reims-Courcy (5\% en cumul) avec un biais froid moyen de $2^{\circ} \mathrm{C}$. Les fréquences égales à $-2^{\circ} \mathrm{C}$ sont considérées comme extrêmes pour la vigne car elles suscitent le gel agronomique même si elles ne font pas partie des extrêmes statistiques inférieures au $5^{\text {ème }}$ centile.

\subsection{Synthèse critique}

Les extrêmes tels que les gelées printanières sont le résultat d'une combinaison de paramètres météorologiques difficiles à modéliser qui résultent de divers processus (advectif, radiatif ou combinés) et qui sont fortement tributaires de la couche nuageuse. Les modèles climatiques actuellement disponibles ne disposent pas d'une résolution spatio-temporelle suffisamment fine pour fournir des simulations précises des extrêmes. Les résultats obtenus lors de cette comparaison corroborent les conclusions en termes d'extrêmes : les modèles ne simulent pas encore correctement les extrêmes car leur distribution spatiale se rapproche moins de la réalité (Terray et Braconnot, 2007). De plus, les différences entre les données simulées représentatives de mailles de 50 ou $300 \mathrm{~km}$ de côté et les données ponctuelles d'une station de référence font partie des marges d'incertitude de ce type d'étude: une maille modélise plus de paramètres spatiaux que la station retenue. Dans ce contexte, le modèle ARPEGE-Climat (Retic), dédié aux études d'impacts du changement climatique aux échelles régionales de la France semble plus adapté à l'étude sur le vignoble marnais et le biais froid peut être en partie corrigé par une prise en compte de l'altitude. Les variations d'altitude entre les points de grille du modèle ARPEGE-Climat (Retic) et la station de référence ReimsCourcy expliquent en partie (environ $1{ }^{\circ} \mathrm{C}$ ) ce biais froid.

\section{Evolution possible des températures extrêmes pour les différents scénarios de simulations futures 2001-2100 pour les deux modèles retenus}

Les scénarios futurs permettent d'obtenir un aperçu de l'évolution des extrêmes climatiques, en tenant compte des biais inhérents aux sorties de modèles et des marges d'incertitude, au sein du vignoble de la Marne. Ils peuvent aider à anticiper les conséquences d'une nouvelle donne climatique au sein du vignoble marnais et surtout, aider la profession viticole à s'adapter à des évènements extrêmes certainement plus fréquents. Ces extrêmes sont analysés par décennie afin de lisser la forte variabilité interannuelle que produisent les deux modèles sur la période 2001-2100.

\subsection{Extrêmes chauds estivaux probables}

Les simulations (figure 4) des scénarios A2 et A1B du modèle ARPEGE-Climat (Retic) prévoient, sur la période future, des hausses des extrêmes estivaux plus importantes que pour le modèle LMD. Pour le scénario B1, le plus optimiste, les fréquences prévues par les deux

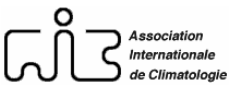


modèles sont très proches jusqu'à la décennie 2071-2080, avec une hausse rapide pour le modèle ARPEGE-Climat (Retic).

Figure 4 : Evolution décennale future des extrêmes estivaux (JJA) supérieurs ou égaux à $35^{\circ} \mathrm{C}$ de 2001 à 2100 pour les deux modèles étudiés (point D ARPEGE-Climat et point LMD) et pour les trois scénarios de simulations (données : Météo-France - CNRM ; IPSL - LMD). Future evolution decades of summer extremes (JJA) upper or equal to $35^{\circ} \mathrm{C}$ from 2001 to 2100 for two studied models (D point for ARPEGE-Climate model and the LMD point) and for three future simulations (data from Météo-France - CNRM ; IPSL - LMD).

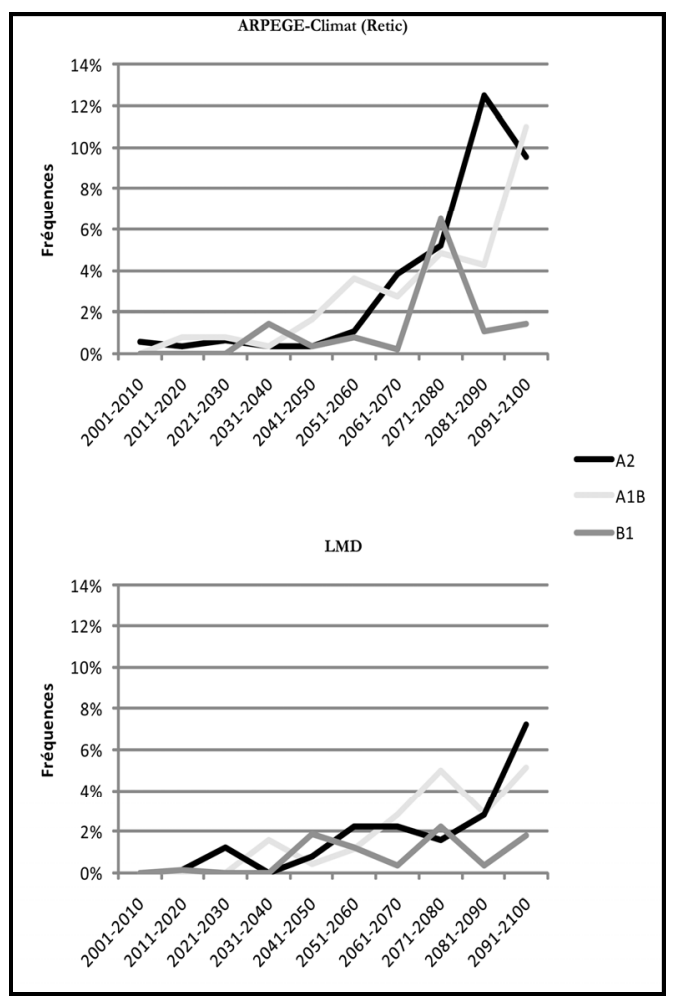

\subsection{Extrêmes froids printaniers probables}

Les fréquences (figure 5) simulées pour les extrêmes froids printaniers futurs présentent des tendances générales à la baisse pour les deux modèles.

Figure 5: Evolution décennale future des extrêmes printaniers (avril) supérieurs ou égaux à $-2^{\circ} \mathrm{C}$ de 2001 à 2100 pour les deux modèles étudiés (point D ARPEGEClimat et point LMD) et pour les trois scénarios de simulations (données: Météo-France -CNRM; IPSLLMD). Future evolution decades of spring extremes lower or equal to $-2^{\circ} \mathrm{C}$ (April) from 2001 to 2100 for two studied models (D point for ARPEGE-Climate model and the LMD point) and for three future simulations (data from MétéoFrance-CNRM ; IPSL - LMD).

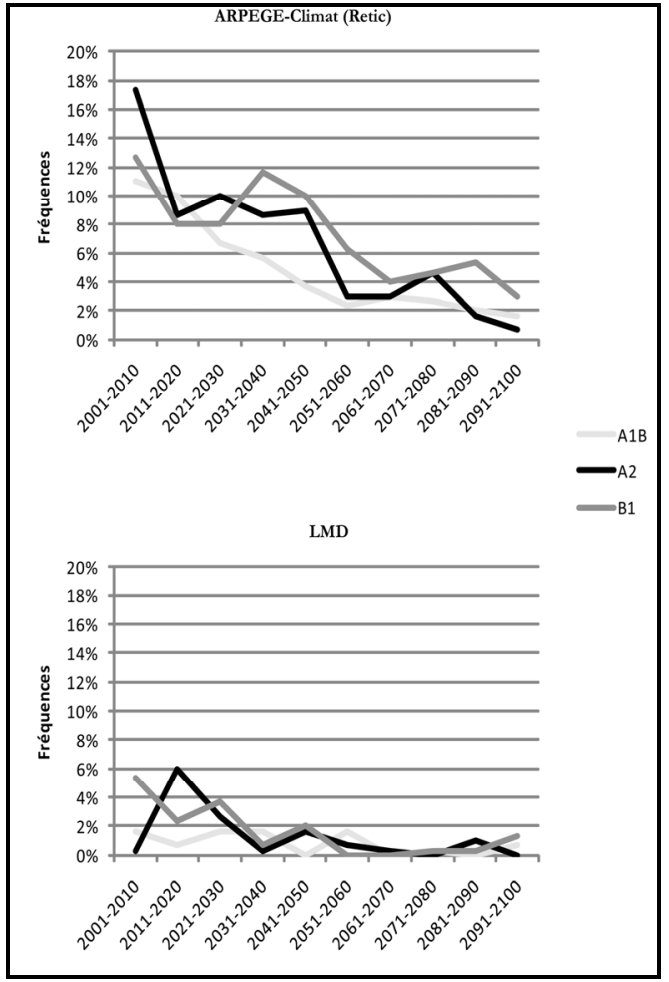


Pour le modèle ARPEGE-Climat, la baisse la plus élevée sur la période future est observée pour le scénario le plus chaud (A2) et la moins importante pour le scénario B1. Les fréquences futures prévues par le modèle LMD présentent une baisse sur la période mais cette fois, le scénario A1B a la diminution la plus faible : la logique des scénarios n'est pas évidente. Des baisses importantes des extrêmes froids printaniers signifient que le vignoble marnais pourrait être moins soumis dans le futur aux aléas gélifs en avril ; cependant, la hausse des températures pourrait favoriser un débourrement plus précoce, en l'avançant en mars. Pour ce mois, le modèle ARPEGE-Climat (non montré) annonce une légère hausse des fréquences d'aléas gélifs pour le scénario B1, tandis qu'on remarque cette même caractéristique pour le scénario A2 du LMD. Cette légère hausse pour le modèle ARPEGEClimat pourrait conduire à augmenter les dommages en cas de débourrement plus précoce.

\section{Conclusion}

L'analyse des sorties de modèles, notamment celles d'ARPEGE-Climat (Retic) à $50 \mathrm{~km}$ de résolution englobant le vignoble de Champagne valident les conclusions du GIEC : i) les fréquences des extrêmes chauds estivaux vont augmenter dans le futur, ii) celles des extrêmes froids printaniers diminuer en avril mais pourront augmenter en mars selon les scénarios et iii) la période future témoigne d'une forte variabilité interannuelle. Même si les modèles ne sont pas encore capables de simuler avec précision les interactions complexes à l'origine des températures extrêmes, l'étude montre que les sorties de modèles ARPEGE-Climat (Retic) simulent correctement l'allure des distributions de températures journalières sur les périodes clés étudiées de la station Reims-Courcy (1950-2000). Ces sorties ARPEGE-Climat (Retic) s'avèrent plus adaptées que le modèle du LMD pour ce type d'étude en raison de leur résolution et d'une meilleure prise en compte de l'altitude. Cependant, des biais subsistent quant à l'estimation des extrêmes thermiques : le modèle témoigne d'un biais froid évident et tend à accentuer les températures extrêmes minimales printanières. Ce type d'étude, au carrefour entre les sciences de la modélisation climatique et de l'agronomie, met en évidence les limites inhérentes à l'utilisation de sorties de modèles issues de sorties de MCG. Malgré les incertitudes qui en résultent, il apparaît une tendance robuste à l'augmentation de la fréquence probable d'aléas extrêmes dommageables pour le cycle végétatif et la récolte, qui se superposera à l'évolution lente du climat moyen. La disponibilité de projections climatiques avec des résolutions spatiales fines est donc une nécessité de plus en plus forte pour préparer les mesures d'adaptation nécessaires dans le vignoble.

Remerciements : Les auteurs remercient D. MONCOMBLE et F. LANGELLIER (Comité Interprofessionnel du Vin de Champagne) pour l'obtention des données climatiques ainsi que S. SOMOT (Météo-France) pour les sorties de modèle ARPEGE-Climat et H. LE TREUT pour les sorties du modèle LMD (IPSL). Ce travail est réalisé dans le cadre du projet Jeune Chercheur ANR 'TERVICLIM' coordonné par H. QUENOL.

\section{Bibliographie}

BENISTON M., STEPHENSON D. B., CHRISTENSEN O. B., FERRO C. A. T., FREI C., GOYETTE S., HALSNAES K., HOLT T., JYLHA K., KOFFI B., PALUTIKOF J., SCHOLL R., SEMMLER T. et WOTH K., 2007 : Future extreme events in European climate: an exploration of regional climate model projections. Climatic Change, 81, 71-95.

BINDI M. et HOWDEN M., 2004 : Challenges and opportunities for cropping systems in a changing climate. Proceedings of the 4th International Crop Science Congress, Brisbane (Australia), 1-13.

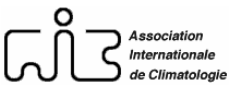


CHAMPAGNOL F., 1984 : Éléments de Physiologie de la vigne et de viticulture générale. Saint-Gely-du-Fesc, France, 351 pages.

DEQUE M. et PIEDELIEVRE J.-P., 1995 : High-Resolution climate simulation over Europe. Climate Dynamics, 11, 321-339.

DOKOOLZIAN N. K. et BERGQVIST J. A., 2001 : Influence of sunlight exposure on the berry growth and composition of two reds cultivars. In Proceedings of the 12th GESCO Conference (Montpellier), 1, 77-84.

DUFRESNE J.-L. et ROYER J.-F., 2007 : Simulation de l'évolution du climat aux échelles globales et régionales. Congrès SHF-29 Journées de l'Hydraulique : "Variations climatiques et hydrologie", Lyon, 14 pages.

DUFRESNE J.-L., SALAS Y MELIA D., DENVIL S., TYTECA S., ARZEL O., BONY S., BRACONNOT P., BROCKMANN P., CADULE P., CAUBEL A., CHAUVIN F., DEQUE M., DOUVILLE H., FAIRHEAD L., FICHEFET T., FOUJOLS M.-A., FRIEDLINGSTEIN P., GRANDPEIX J.-Y. et GUEREMY J.-F., 2006 : Simulation du climat récent et futur par les modèles du CNRM et de l'IPSL. La Météorologie, 55, 45-59.

GARCIA DE CORTAZAR A. I., 2006 : Adaptation du modèle STICS à la vigne (/Vitis vinifera/ L.), Utilisation dans le cadre d'une étude du changement climatique à l'échelle de la France. Thèse de doctorat, Ecole Nationale Supérieure Agronomique, Montpellier, 292 pages.

GIBELIN A.-L. et DEQUE M., 2003 : Anthropogenic climate change over the Mediterranean region simulated by a global variable resolution model. Climate Dynamics, 20, 327-339.

GIEC, 2001 : Climate Change 2001: impacts, adaptation and vulnerability. Contribution of Working Group II to the third assessment report of IPCC, Cambridge University Press, Cambridge, 89 pages.

GIEC, 2007 : Climate Change 2007: the AR4 Synthesis Report. Edited by Rajendra K. Pachauri, IPCC Chairman, Andy Resinger, Head of Technical Support Unit, The Core Writing Team, IPCC, Geneva, Switzerland, 114 pages.

HUGLIN P. et SCHNEIDER C., 1998 : Biologie et écologie de la vigne. Paris, Editions Payot Lausanne, TEC et DOC, 371 pages.

JONES G. V., DUCHENE E., TOMASI D., YUSTE J., BRASLAVSKA O., SCHULTZ H., MARTINEZ C., BOSO S., LANGELLIER F., PERRUCHOT C. et GUIMBERTEAU G., 2005a : Changes in European winegrape phenology and relationships with climate. GESCO, 55-62.

JONES G. V., WHITE M. A., COOPER O. R. et STORCHMANN K., 2005b : Climate and Wine: quality issues in a warmer world. Climatic change, 73-3, 319-343.

JONES G. V., 2006 : Climate and Terroir: Impacts of Climate Variability and Change on Wine. In Fine Wine and Terroir - The Geoscience Perspective. Macqueen R.W. and Meinert L.D., Geoscience Canada Reprint Series Number 9, Geological Association of Canada, St. John's, Newfoundland, 247 pages.

JONES G. V., 2007 : Climate Change: Observations, Projections, and General implications for viticulture and wine production. In Global Warming, which potential impacts on the vineyards?, UNESCO Chair Wine and Culture, Dijon (28-30 mars), 15 pages.

KHARIN V. V. et ZWIERS F. W., 2000 : Changes in the extremes in an ensemble of transient climate simulations with a coupled Atmosphere-Ocean GCM. Journal of Climate, $3760-3788$. 
LAUFFENBURGER M., 2007 : Estimation du réchauffement climatique dans le sud-est de la France (à l'est du Rhône) à la fin du XXIème siècle (2071-2100). Thèse de doctorat de l'Université Aix-Marseille 1, 294 pages.

MADELIN M., 2004 : L'aléa gélif printanier dans le vignoble marnais en Champagne : modélisation spatiale aux échelles fines des températures minimales et des écoulements de l'air. Thèse de doctorat de l'université Paris 7, 353 pages.

MATUS J. T., LOYOLA R., VEGA A., PENA-NEIRA A., BORDEU E., ARCE-JOHNSON P. et ALCADE J. A., 2009: Post-veraison sunlight exposure induces MYB-mediated transcriptional regulation of anthocyanin and flavonol synthesis in berry skins of Vitis Vinifera. Journal of experimental botany, 60-3, 853-867.

MOISSELIN J-M., SCHNEIDER M., CANELLAS C. et MESTRE O., 2002 : Les changements climatiques en France au $\mathrm{XX}^{\text {ème }}$ siècle. Étude des longues séries de données homogénéisées françaises de précipitations et températures. La Météorologie, 38, 45-46.

NAKICENOVIC N. et SWART R., 2000 : Rapport spécial du GIEC, Scénarios d'émissions, Résumé à l'intention des décideurs, 27 pages.

PALUTIKOF J. P., WINKLER J. A., GOODNESS C. M. et ANDRESEN J. A., 1997 : The simulation of daily temperature time series from GCM output. Part 1: Comparison of model data with observation. Journal of Climate, 10, 2497-2513.

PETTITT A. N., 1979 : A non-parametric approach to the changepoint problem. Applied Statistics, 28-2, 126-135.

SALAS-MELIA D., CHAUVIN F., DEQUE M., DOUVILLE H., GUEREMY J. F., MARQUET P., PLANTON S., ROYER J.-F. et TYTECA S, 2005 : Description and validation of the CNRM-CM3 global coupled model. Note de centre $n^{\circ} 103 d u$ CNRM, Toulouse, Météo-France, 36 pages.

SEGUIN B., 2004 : Le réchauffement climatique récent en France : impact et conséquence sur la culture des arbres fruitiers et de la vigne. Lettre ${ }^{\circ} 16$ PIGB-PMRC, http://www.cnrs.fr/cw/dossiers/dosclim/biblio/pigb16/09_rechauffement.htm.

SIEGEL S. et CASTELLAN N. J., 1988 : Nonparametric Statistics for the Behavioural Sciences. Mc Graw-Hill, New York, 399 pages.

STEPHENS M. A., 1974 : EDF Statistics for Goodness of Fit and Some Comparisons. Journal of the American Statistical Association, 69, 730-737.

TERRAY L. et BRACONNOT P., 2007 : Livre blanc ESCRIME, Etude des simulations climatiques. Dans le cadre du projet ESCRIME, édité avec le soutien de l'INSU, de l'ONERC et de l'Iddri, 70 pages.

WILLIAMS D., 2001 : Weighing the Odds: a Course in Probability and Statistics. Cambridge University Press, 548 pages. 